\title{
Target Lesions and Other Paintball Injuries
}

\author{
Jennifer A. Sbicca, BS, and Robert L. Hatch, MD, MPH
}

Paintball is a popular combat game, with more than $\mathbf{5}$ million participants per year. As it has increased in popularity, the incidence of paintball-related injuries also has increased. The most common injuries are classic, benign skin lesions that are easily recognized if one is aware of them. Devastating eye injuries also may occur if participants do not wear face masks. Other reported injuries include musculoskeletal injuries, solid organ injuries, and vascular pseudoaneurysms. Rarely, paintball-related deaths have been reported. This article is the first to review the full spectrum of paintball injuries; in addition, the article emphasizes the importance of encouraging participants to adhere to appropriate safety measures, particularly wearing an appropriate face mask at all times during the game. ( $\mathrm{J}$ Am Board Fam Med 2012;25:124-127.)

Keywords: Athletic Injuries, Case Report, Literature Review, Purpura

Paintball is a popular, war-like game in which the players are armed with guns powered by compressed gas that fire paintballs. ${ }^{1}$ In 2007 , an estimated 5,476,000 people in the United States played paintball at least once during the year. ${ }^{2}$ Paintballs are spherical and have a central seam designed to break on impact, leaving paint on the player to indicate a "hit." ${ }^{3-5}$ Although paintballs travel at fairly low speeds and are designed to break easily, they still are capable of causing injury. Concurrent with the rise in popularity of the game, the incidence of paintball-related injuries is increasing. ${ }^{6,7}$

This article describes a patient with a common paintball injury and reviews the literature about paintball injuries. By understanding basic aspects of the game and the injuries associated with it, family physicians can better recognize and treat these injuries, as well as help prevent injuries by educating participants about safety precautions. A literature review was performed by searching PubMed with the following words: paintball, paint, pellet, and air-

This article was externally peer reviewed.

Submitted 13 April 2011; revised 6 June 2011; accepted 15 June 2011.

From the Department of Community Health and Family Medicine, University of Florida, Gainesville, FL.

Funding: none.

Conflict of interest: none declared.

Corresponding author: Robert L. Hatch, Department of Community Health and Family Medicine, College of Medicine, University of Florida, 1600 SW Archer Road, Box 100222, Gainesville, FL 32610 (E-mail: hatch@ufl.edu). gun. The lists of relevant paintball articles were carefully reviewed to identify additional pertinent publications.

\section{Case Report}

A 53-year-old man presented with multiple tender skin lesions (Figure 1). Similar lesions were noted on his trunk and arm. He played paintball 3 days earlier at a commercial site, sustaining multiple hits with paintballs. His medical history was positive only for asthma, which was treated with albuterol metered dose inhaler as needed. He had no history of abnormal bleeding or skin problems. The lesions shown in Figure 1 were caused by shots fired from approximately 40 yards away. Immediately after impact, raised welts appeared. These evolved into bruises over the next 12 to 24 hours. The lesions on the patient's leg were more dramatic than those on his trunk; he attributed this to wearing only a thin pair of pants but wearing several layers of clothing on his trunk. He had sustained several hits to his face and forehead, but his facemask fully protected these areas from injury. One month after presentation, all skin lesions had resolved without scarring.

\section{Discussion}

Paintballs reach a velocity of 100 to $300 \mathrm{ft} / \mathrm{sec}$ and are filled with a nontoxic, water-soluble, colorful dye. ${ }^{1,2,8,9}$ Because of their large size and relatively 
Figure 1. Skin lesions due to paintball impacts.

Known as paintball purpura, these lesions may have either a "target" shape (2 lesions lower on leg) or an annular shape (lesion near knee).

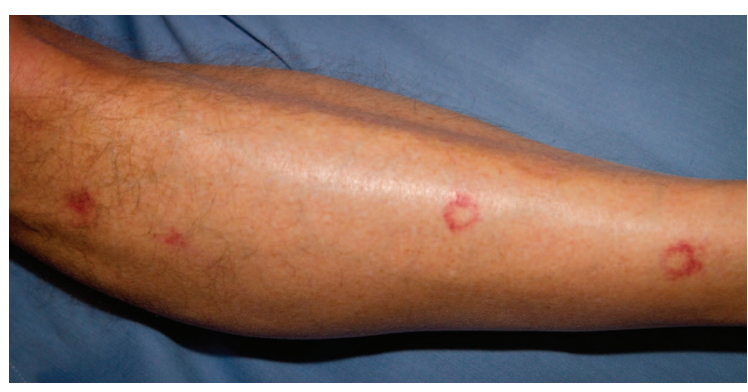

low velocity compared with other airgun projectiles, paintballs cause blunt rather than penetrating injuries. No deaths have been reported as being caused directly by paintballs, ${ }^{9}$ but 2 deaths were caused by high-pressure carbon dioxide canisters that accidentally dislodged from paintball guns. ${ }^{10}$

Paintball games occur at commercial sites, at military training sites, and at private homes. In the United States, commercial sites require participants to wear polycarbonate masks approved by the American Society for Testing and Materials (ASTM) that cover the eyes, nose, mouth, and ears at all times (Figure 2). Many players prefer to play at home, and while playing there they are not required to wear ASTM-certified masks and as a result have a much higher risk for injury. ${ }^{7}$ Physicians who understand paintball can play a role in educating patients about the importance of wearing appropriate face protection at all times while playing. ${ }^{11}$

Paintball has been associated with a wide range of injuries (summarized in Table 1). Most of the literature describing paintball injuries is from case studies and emergency department and ophthalmology registries. ${ }^{6,12,13}$ Aside from skin lesions and musculoskeletal injuries, which are discussed below, ocular injuries are the most common, and many require urgent surgical intervention. ${ }^{6,13}$ The range of reported ocular injury includes hyphema, ${ }^{11-16}$ minor ocular trauma, such as corneal abrasion ${ }^{11,12}$ and paint in the eye,${ }^{17}$ to open globe ruptures, ${ }^{11,12,15}$ and loss of an eye. ${ }^{12}$ The visual prognosis for ocular injuries is poor. Thirty percent to $43 \%$ of patients with ocular injuries become legally blind in the injured eye (visual acuity $\leq 20$ / 200). ${ }^{11,12,14}$ In experimental simulations, paintballs traveling as slow as $65 \mathrm{ft} / \mathrm{sec}$ can cause globe rupture, and the risk of globe rupture nears 100\% when paintballs travel faster than $98 \mathrm{ft} / \mathrm{sec}^{4}$ Farr and Fekrat ${ }^{12}$ reviewed 87 cases of worldwide ocular paintball injuries in 1999 and found that the 3 most common injuries were hyphemas $(n=69)$, vitreous hemorrhages $(\mathrm{n}=37)$, and traumatic cataracts $(\mathrm{n}=$ 31). ${ }^{12}$ Listman et $\mathrm{al}^{11}$ reviewed 149 cases of pediatric paintball ocular injuries in 2004 and found 149 hyphemas, 50 vitreous hemorrhages, and 42 traumatic cataracts. Most of the ocular injuries occurred in young men. ${ }^{6,11,12}$

There were no cases in the literature in which a patient sustained an ocular injury while wearing a mask approved by the ASTM. ${ }^{14}$ Most participants experienced ocular injury when masks were not worn or when masks were removed temporarily because of paint or fogging. ${ }^{9,11,12,15,16}$ There are a few case reports of paintballs dislodging masks or the paintball traveling under goggles. ${ }^{9,14,15}$ The patients in these reports did not wear a mask approved by the ASTM for paintball games. ${ }^{14}$

Almost all people who play paintball develop purpuric lesions at the site of paintball impact, making it the most common injury sustained while playing paintball. Five articles detail the dermatologic manifestations of paintball, and the term paintball purpura has emerged as the preferred terminology. ${ }^{3,8,18-20}$ Their unusual shape may initially confound diagnosis, and the differential diagnosis for an annular erythema includes cupping,

Figure 2. A paintball player wearing American Society for Testing and Materials-approved face mask. Though the mask does an excellent job of protecting the face, eyes, nose, and mouth, it offers little or no protection to the forehead and scalp. This explains the occasional occurrence of traumatic scalp pseudoaneurysms among paintball players.

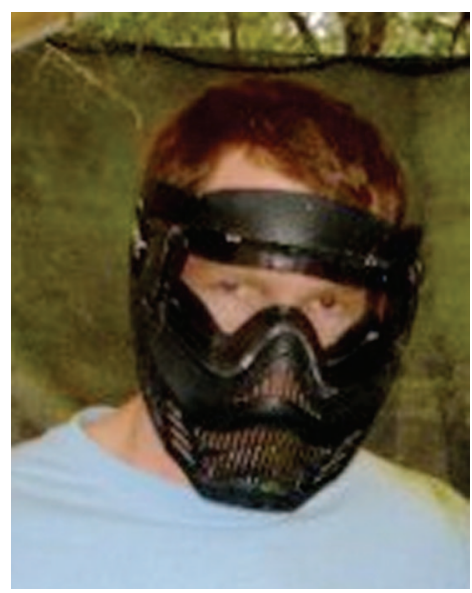


Table 1. Summary of Reported Paintball Injuries

\begin{tabular}{ll}
\hline Injury & Reference in Which Injury Was Reported \\
\hline Paintball purpura & Scott \& Scott, 1989 (21) \\
$\begin{array}{l}\text { Nonspecific sports injury (strain/sprain, contusion/abrasion, laceration, fracture) } \\
\text { Ocular injury (hyphema, vitreous hemorrhage, traumatic cataract) }\end{array}$ & Conn et al, 2004 (6) \\
& Listman, 2004 (11) \\
Traumatic pseudoaneurysm & Farr \& Fekrat, 1999 (12) \\
Solid organ injury & Cohen \& Itshayek, 2010 (23) \\
$\quad$ Renal & \\
$\quad$ Urologic & Guerrero et al, 2009 (27) \\
& Agrawal et al, 2006 (25) \\
\hline
\end{tabular}

erythema perstans, insect bite reactions, erythema annulare centrifugum, erythema mutliforme, granuloma annulare, tinea corporis, Majocchi disease (purpura annularis telangiectodes), gyrate erythemas, physical abuse, and ping pong ball purpura. ${ }^{5,18,20,21}$ The uniform size of the lesions, lack of associated patient symptoms, and history of paintball participation usually excludes the other annular erythemas. ${ }^{20}$ Paintball purpura generally resolves over 1 to 2 weeks with no residual deficits, although one case reported the development of an annular hypopigmented scar. ${ }^{8}$ Accurate diagnosis of paintball purpura avoids unnecessary testing and provides an opportunity to discuss paintball safety measures. ${ }^{22}$

Conn et al's $\mathrm{s}^{6}$ review of patients with paintball injuries who presented to an emergency department reminds us that paintball is a sport and, like other sports, participants may experience nonspecific sports-related injuries. The game of paintball involves running, rapid direction change, and attempts to dodge paintballs. Eighteen percent of emergency room visits secondary to paintball activity involved overexertion and falls. Ankle and knee sprains were especially common, and fractures and lacerations were observed as well.

Though ocular, dermatologic, and sports-related injuries are the most common injuries sustained during paintball, paintballs can cause other, less common injuries as well. Three case reports detail traumatic pseudoaneurysm of the scalp arteries caused by blunt paintball injury. ${ }^{5,23,24}$ During physical examination, these lesions were pulsatile and cystic. ${ }^{23}$ The differential diagnosis for this type of lesion includes inclusion cyst, hematoma, abscess, arteriovenous fistula, and lipoma ${ }^{5,23}$; however, physical examination findings of a palpable thrill and a history of paintball participation excludes other diagnoses. The lesions are unattractive but not medically dangerous to the individual. Treatment options include endovascular occlusion, surgical excision, manual compression, and thrombin injection. ${ }^{23}$

Solid organ injury secondary to a paintball was reported in three cases. ${ }^{25-27}$ One case of subcapsular hematoma of the kidney secondary to paintball injury was reported..$^{27}$ The patient presented with hematuria and flank pain after paintball play and was diagnosed by computed tomography. The patient's symptoms resolved in 4 days and the subcapsular hematoma was resolved at 9 months. Two cases of urologic trauma also have been reported (scrotal rupture and penoscrotal lymphedema), and both cases required surgical correction. ${ }^{25,26}$ The patient with scrotal rupture presented to a family physician, requiring prompt recognition of this urologic emergency. Acute myocardial infarction while playing paintball also has been reported. ${ }^{28}$ The patient, a 25 -year-old male professional paintball player, had thrombosis in the left anterior descending artery at the site of a spontaneous coronary artery dissection, which was attributed to extreme physical exertion.

Awareness of the popularity and hazards of paintball and the ability to identify the characteristic skin lesions provides an opportunity for the family physician to discuss appropriate paintball safety precautions with patients. Increased risk of injury is associated with young men, playing at noncommercial settings, and lack of appropriate eye protection. ${ }^{11}$ Identifying where the patient is playing paintball is a crucial part of the discussion because a rising percentage of ocular injuries occur at home sites. ${ }^{11,14}$ If paintball is played at home sites, children should be supervised and required to wear appropriate masks at all times. In Florida, where this article was written, state law makes it a second-degree misdemeanor for a minor younger than 16 years of to use a BB gun, air gun, 
or gas-operated gun unless an adult is supervising the use and the minor's parent has consented to the use. Family physicians also can focus their guidance on the use of appropriate face masks. ASTM-approved face masks with polycarbonate lenses are extremely effective in preventing eye injuries during paintball games. ${ }^{14}$ The patient should be instructed never to take off the face mask during play under any circumstances. If a patient reports playing without a mask because of fogging of the mask, the availability of antifog inserts and solutions should be discussed. In addition, strips of peelable plastic can be placed on the front of the mask and peeled off when paint obstructs vision instead of taking off masks to wipe paint away. Patients should be advised to wear appropriate protective clothing to minimize injuries and strictly advised to avoid shooting anyone at close range $(<10$ yards).

\section{References}

1. Bellis M. Paintball: history-guns and markers. Available at http://inventors.about.com/library/ inventors/blpaintball.htm. Accessed February 19, 2011.

2. SGMA. Extreme sports: an ever-popular attraction. July 07, 2008. Available at http://www.sgma. com/press/2_Extreme-Sports\%3A-An-Ever-PopularAttraction. Accessed March 29, 2011.

3. Levsky ME, Crowe M. What is your diagnosis? Paintball purpura. Cutis 2005;75:157-8.

4. Weaver AA, Kennedy EA, Duma SM, Stitzel JD. Evaluation of different projectiles in matched experimental eye impact simulations. J Biomech Eng 2011;133:031002.

5. Fox JT, Cordts PR, Gwinn BC. Traumatic aneurysm of the superficial temporal artery: case report. J Trauma 1994;36:562-4.

6. Conn JM, Annest JL, Gilchrist J, Ryan GW. Injuries from paintball game related activities in the United States, 1997-2001. Inj Prev 2004;10:139-43.

7. SGMA. Sanctioned Team Sports Play In the US Remains Strong, But ... . October 12, 2008. Available at http://www.sgma.com/press/93_SanctionedTeam-Sports-Play-In-the-US-Remains-Strong,-But. Accessed March 29, 2011.

8. Ambay AR, Stratman EJ. Paintball: dermatologic injuries. Cutis 2007;80:49-50.

9. Laraque D, American Academy of Pediatrics Committee on Injury, Violence, and Poison Prevention. Injury risk of nonpowder guns. Pediatrics. 2004;114: $1357-61$.
10. CPSC. CPSC issues new safety warning for paintball guns. March 24, 2004. Available at http://www.cpsc.gov/ CPSCPUB/PREREL/prhtml04/04105.html. Accessed January 20, 2011.

11. Listman DA. Paintball injuries in children: more than meets the eye. Pediatrics 2004;113:15-8.

12. Farr AK, Fekrat S. Eye injuries associated with paintball guns. Int Ophthalmol 1999;22:169-73.

13. Kitchens JW, Danis RP. Increasing paintball related eye trauma reported to a state eye injury registry. Inj Prev 1999;5:301-2.

14. Fineman MS. Ocular paintball injuries. Curr Opin Ophthalmol 2001;12:186-90.

15. Thach AB, Ward TP, Hollifield RD, et al. Ocular injuries from paintball pellets. Ophthalmology 1999; 106:533-7.

16. Zwaan J, Bybee L, Casey P. Eye injuries during training exercises with paint balls. Mil Med 1996; 161:720-2.

17. Vassilev ZP, Marcus SM. Paintball injuries in children: the cases managed out of hospitals. Pediatrics 2004; $113: 1468$.

18. Aboutalebi S, Stetson CL. Paintball purpura. J Am Acad Dermatol 2005;53:901-2.

19. Siegel DM, Goldberg LH, Altman AR, Kalter DC. Paint pellet purpura: a peril for pistol-packing paramilitary personnel. JAMA 1986;255:3367.

20. Rahbari H, Nabai H. Paint pellet erythema. Pediatr Dermatol. 1996;13:174-5.

21. Scott MJ Jr, Scott MJ III. Ping pong patches. Cutis 1989;43:363-4.

22. Bender TW. Cutaneous manifestations of disease in athletes. Skinmed 2003;2:34-40.

23. Cohen JE, Itshayek E. Traumatic pseudoaneurysm of the superficial temporal artery after paintball injury. Isr Med Assoc J 2010;12:123-4.

24. John N, Leach JL, Rachana T, Mangano FT. Traumatic aneurysm of the occipital artery secondary to paintball injury. Clin Neurol Neurosurg 2009;111: 105-8.

25. Agrawal V, Li C, Minhas S, Ralph D. Paint ball injury resulting in penoscrotal lymphedema. Urology 2006;67:1288-9.

26. Joudi JN, Lux MM, Sandlow JI. Testicular rupture secondary to paint ball injury. J Urol 2004;171:797.

27. Guerrero MA, Zhou W, El Sayed HF, Kougias P, Lin PH. Subcapsular hematoma of the kidney secondary to paintball pellet injuries. J Emerg Med 2009;36:300-1.

28. Shah SH, Sketch MH Jr, Tcheng JE. Acute myocardial infarction in a young male while playing paintball. J Invasive Cardiol 2002;14:713-5. 